\title{
Optimal Solution of Structural Engineering Design Problems using Crow Search Algorithm
}

\author{
Madhur Agarwal \\ Department of Electronics \& Communication Engineering \\ Thapar Institute of Engineering and Technology \\ Deemed to be University, Patiala, Pin -147004, Punjab, India \\ Email-agarwalmadhur27@gmail.com
}

(Received November 15, 2018; Accepted April 23, 2019)

\begin{abstract}
In real world, the structural engineering design problems are large scale non-linear constrained problems. In the present study, crow search algorithm (CSA) is applied to find the optimal solution of structural engineering design problems such as pressure vessel design problem, welded beam design problem and tension/ compression string design problem. The numerical results are compared with the existing results reported in the literature including metaheuristic algorithms and it is found that the results obtained by the crow search algorithm are better than other existing algorithms. Further, the effectiveness of the algorithm is verified to be better than the existing algorithms by statistical analysis using mean, median, best case, and worst case scenarios. The present study confirms that the crow search algorithm may be easily and effectively applied to various structural design problems.
\end{abstract}

Keywords- Metaheuristic algorithm, Crow search algorithm, Constrained optimization.

\section{Introduction}

In everyday life, we come across various problems for which we need to find the optimum solution. Design optimization is the process of finding the optimal values of various parameters defining the problem, which provides the best value of the objective function. Depending upon the problem, the objective function can be as simple as a single variable linear function to very complex multi variable non-linear function. Generally, the objective function is accompanied by various constraints related to the nature of the problems. The number of decision variables and constrains can vary from a very small number to a very large number.

Mathematically, the most general optimization problem can be defined as:

Maximize/Minimize $f(\vec{X})$ where $\vec{X}=\left(x_{1}, x_{2}, x_{3}, \ldots, x_{n}\right)$

subject to: $g_{k}(\vec{X}) \geq 0, k=1,2,3, \ldots, m$ and $h_{j}(\vec{X})=0, j=1,2,3, \ldots, p$.

where $f, g$ and $h$ are non-linear real valued functions.

Structural design problems include designing a product in most optimal way such that it satisfies given constraints. There can be a large number of feasible solutions for the given problem and an appropriate optimization technique is used to find the most optimal solution. Though some of these problems can be solved using traditional methods but a lot of problems are non-linear and include mixed design variables under complex constraints, due to which traditional methods fail (Michalewicz, 1994). 
International Journal of Mathematical, Engineering and Management Sciences

Vol. 4, No. 4, 968-981, 2019

https://dx.doi.org/10.33889/IJMEMS.2019.4.4-077

For such problems, Metaheuristic algorithms (Lee and Geem, 2005), which have been found effective for global optimization, are applied (Yang, 2011). These Metaheuristic algorithms generally have their inspiration from processes in nature (Holland, 1975; Glover, 1986). These algorithms have been able to overcome the drawbacks and restrictions faced by traditional numerical methods. Some of the examples of Metaheuristic algorithms developed and used in past have been, Genetic Algorithms (GA) (Michalewicz, 1994; Deb, 2000; Coello and Montes, 2002), Particle Search Optimization (PSO) (Cagnina et al., 2008; Dos Santos Coelho, 2010; Kennedy and Eberhart, 1995; Rao, 1996), Cuckoo Search (Yang and Deb, 2009), Harmony Search (Geem et al., 2001). A lot of people have developed and applied various Metaheuristic algorithms to such structural design problems. Many authors such as Deb (1991), Coello (2000), Dimopoulos (2007), Hwang and He (2006) used Genetic Algorithms to solve various problems. An advanced version of differential evolution algorithms have been applied by Montes et al. (2007). Particle Search Optimization (PSO) has been used by Shi and Eberhart (1998), He et al. (2004). Moreover, Harmony Search algorithms have been used by Lee and Geem (2005) for solving design optimization problems. Artificial bee colony algorithm has been used by Garg (2014) for solving structural engineering design optimization problem. Further, two challenging engineering design problems are solved using salp swarm algorithm by Mirjalili et al. (2017). In the present study, nature inspired algorithm known as the crow search algorithm (Zolghadr and Bozorg, 2018) has been applied for solving the structural engineering design problems. Crows are considered one of the intelligent organisms who can remember things. Crows are also smart; they can fool other crows who are following them by deviating from their paths and following a new path but at the same time remembering their previous path. These characteristics of crows have been utilized to search for the next optimal solution while remembering the previous best solution and in this way reaching the global optimal solution.

The present paper is organized as follows: in Section 2, the crow search algorithm is presented. In Section 3, the mathematical model of three structural engineering design problems and their numerical results are presented. The paper closes with conclusion in Section 4.

\section{Algorithm}

The crow search algorithm (CSA) is a metaheuristic optimization algorithm. This algorithm was initially introduced by Askarzadeh (2016). It is based on intelligent behavior of crow flocks. CSA attempts to imitate the social intelligence of crow flock and their food gathering process. A crow individual has a tendency to tap in to the food resources of other species, including the other crow members of the flock. In fact each crow attempts to hide its excess food in a hideout spot and retrieve the stored food in the time of need. However the other members of the flock, which have their own food reservation spots as well, try to tail one another to find these hidings spots and plunder the stored food. In the standard CSA, the flock of crows spread and search throughout the decision space for the perfect hideout spots (global optima). Subsequently, each crow individual shall make a motion based on the two basic principles of the CSA: (i) protecting its own hideout spot and (ii) detecting the other members' hideout spot (Zolghadr and Bozorg, 2018). The stepwise procedure is given below:

(i) Define decision variables, objective function and constraints.

(ii) Define adjustable parameters. The adjustable parameters include flock size $(N)$, maximum number of iterations (maxit), flight length $\left(f_{1}\right)$ and awareness probability $(A P)$. 
International Journal of Mathematical, Engineering and Management Sciences

Vol. 4, No. 4, 968-981, 2019

https://dx.doi.org/10.33889/IJMEMS.2019.4.4-077

(iii) Initialize the position of crows. Using random function, $N$ crows are randomly positioned in a $d$ dimensional search space. Each crow denotes a feasible solution of the problem and $d$ is the number of decision variables.

(iv) Initialize and record the memory of each crow. Since at the initial iteration, the crows have no experience, it is assumed that they have hidden their food at their initial position.

(v) Check the feasibility of the initial locations generated. Check if they satisfy constraints. Reinitialize the locations till it reaches a set of locations that satisfy the constraints giving a feasible solution.

(vi) Randomly select one crow (called $j$ ), and other crows would follow this crow to update their locations using equation

$X_{(i, i t e r+1)}=X_{(i, i t e r)}+r_{(i)} \times f_{1(i, i t e r)} \times\left(m_{(j, i t e r)}-x_{(i, i t e r)}\right)$ if $r(j) \geq A P(i$, iter $)$.

Else generate a random (position) feasible solution in search space if $r(j)<A P(i$, iter $)$, where $r(j)$ is a random number with uniform distribution between 0 and 1 and $A P(i$, iter $)$ denotes the awareness probability of crow $j$ at iteration iter.

(vii) Check the feasibility of the new positions of each crow in decision space. If not feasible, generate a feasible random solution.

(viii) If the new position is feasible, the crow updates its position. Otherwise, the crow stays in the current position and does not move to the new generated position.

(ix) Evaluate the new position of the crows. The fitness function value for the new position of each crow is computed. If the fitness (objective function) value of the new position is better than the fitness function value of the memorized position, the crow updates its memory by new position.

(x) Reiterate till maximum number of input iterations. When termination criterion is satisfied, the best position of the memory in terms of the objective function value is reported as the solution of the optimization problem.

\section{Structural Optimization Problems}

The crow search algorithm has been applied on various structural engineering design problems, including their different versions.

\subsection{Design of Pressure Vessel}

It consists of a compressed air storage tank having a volume of $750 \mathrm{ft}^{3}$ and pressure of $2000 \mathrm{psi}$. Hemispherical heads are placed at both ends. The cylindrical shape is formed by two halves of longitudinal welds. We have to minimize the total cost, which includes the cost of the material, forming process and welding process (Kannan and Kramer, 1994). The variables in the problem are thickness of pressure vessel $x_{1}$, thickness of head $x_{2}$, inner radius $x_{3}$ of the vessel and the length $x_{4}$ of the vessel excluding the head.

The fitness function is given by:

$$
\begin{aligned}
& \text { Minimize } f(X)=0.6224 x_{1} x_{2} x_{3}+1.7781 x_{2} x_{3}^{2}+3.1661 x_{1}^{2} x_{4}+19.84 x_{1}^{2} x_{3} \\
& \text { subject to: } \\
& g_{1}(X)=-x_{1}+0.0193 x_{3} \leq 0, g_{2}(X)=-x_{2}+0.00954 x_{3} \leq 0, \\
& g_{3}(X)=-\pi x_{3}^{2} x_{4}-(4 / 3) \pi x_{3}^{3}+1296000 \leq 0, g_{4}(X)=x_{4}-240 \leq 0 .
\end{aligned}
$$


International Journal of Mathematical, Engineering and Management Sciences

Vol. 4, No. 4, 968-981, 2019

https://dx.doi.org/10.33889/IJMEMS.2019.4.4-077

Version 1: Design of pressure vessel problem has been solved by various authors in the following bounded region:

Search Space: $1 \times 0.0625 \leq x_{1}, x_{2} \leq 99 \times 0.0625 ; 10 \leq x_{3}, x_{4} \leq 200$.

The optimal solution in this bounded region using the crow search algorithm is: $X=(0.7784145664935,0.3847801710354,40.3319303998310,199.8303931318342)$

and optimum (minimum) fitness function value: $f(X)=5596.18641830762$.

Constraint values:

$G=\left(g_{1}, g_{2}, g_{3}, g_{4}\right)=(-0.53319600,-2.33772754,-20516404.40392017,-118.87315251)$.

The result obtained using the proposed algorithms are better than any of the existing results. A comparison of the same is given in the Table 1.

\section{Version 2:}

The upper bound of the fourth variable (length of the vessel) is updated to 240 i.e. $10 \leq x_{4} \leq 240$ [Dimopoulos (2007)]. Rest all the things remain the same.

$X=(0.7280931726201,0.3599225433937,37.7241665248285,239.5857630173754)$.

Optimum (minimum) fitness function value: $f(X)=5501.57955042116$.

Constraints value:

$G=\left(g_{1}, g_{2}, g_{3}, g_{4}\right)=(-1.86901631,-2.63361733,-25611830.76570778,-173.16317732)$.

The result for version 2 are also illustrated in Table 1 with comparison to other existing algorithms. Further, statistical analysis has been done and a comparison with other existing algorithms has been shown in the Table 2 .

The comparisons of the simulated results are shown in the Table 1 and the corresponding statistical results are summarized in Table 2 . The results obtained by crow search algorithm are better than any other solutions reported in the literature. 
International Journal of Mathematical, Engineering and Management Sciences

Vol. 4, No. 4, 968-981, 2019

https://dx.doi.org/10.33889/IJMEMS.2019.4.4-077

Table 1. Comparison of the best solution for pressure vessel design problem obtained by different methods

\begin{tabular}{|c|c|c|c|c|c|c|}
\hline \multirow[t]{2}{*}{ Version } & \multirow[t]{2}{*}{ Method } & \multicolumn{4}{|c|}{ Design Variables } & \multirow{2}{*}{$\begin{array}{l}\text { Cost } \\
f(x)\end{array}$} \\
\hline & & $x_{1}$ & $x_{2}$ & $x_{3}$ & $x_{4}$ & \\
\hline \multirow{20}{*}{ I } & Sandgren (1988) & 1.125000 & 0.625000 & 47.700000 & 117.701000 & 8129.1036 \\
\hline & Kannan and Kramer (1994) & 1.125000 & 0.625000 & 58.291000 & 43.690000 & 7198.0428 \\
\hline & $\operatorname{Deb}(1997)$ & 0.937500 & 0.50000 & 48.329000 & 112.67900 & 6410.3811 \\
\hline & Coello (2000) & 0.812500 & 0.437500 & 40.323900 & 200.00000 & 6288.7445 \\
\hline & Coello and Montes (2002) & 0.812500 & 0.437500 & 42.097398 & $176 . .654050$ & 6059.946 \\
\hline & He and Wang (2007) & 0.812500 & 0.437500 & 42.091266 & 176.746500 & 6061.0777 \\
\hline & Montes and Coello (2008) & 0.812500 & 0.437500 & 42.098087 & 176.640518 & 6059.7456 \\
\hline & Kaveh and Talatahari (2009) & 0.812500 & 0.437500 & 42.103566 & 176.573220 & 6059.0925 \\
\hline & Kaveh and Talathari (2010) & 0.812500 & 0.437500 & 42.098353 & 176.637751 & 6059.7258 \\
\hline & Zhang and Wang (1993) & 1.125000 & 0.625000 & 58.290000 & 43.6930000 & 7197.7000 \\
\hline & Cagnina et al. (2008) & 0.812500 & 0.437500 & 42.098445 & 176.6365950 & 6059.714335 \\
\hline & Dos Santos Coelho (2010) & 0.812500 & 0.437500 & 42.098400 & 176.6372000 & 6059.7208 \\
\hline & He et al. (2004) & 0.812500 & 0.437500 & 42.098445 & 176.6365950 & 6059.7143 \\
\hline & Lee and Geem (2005) & 1.125000 & 0.625000 & 58.278900 & 43.75490000 & 7198.433 \\
\hline & Montes et al. (2007) & 0.812500 & 0.437500 & 42.098446 & 176.6360470 & 6059.701660 \\
\hline & He et al. (2003) & 0.812500 & 0.437500 & 42.098450 & 176.6366000 & 6059.131296 \\
\hline & Gandomi et al. (2003) & 0.812500 & 0.437500 & 42.0984456 & 176.6365958 & 6059.7143348 \\
\hline & Akay and Karaboga (2012) & 0.812500 & 0.437500 & 42.098446 & 176.636596 & 6059.714339 \\
\hline & Garg (2014) & 0.7781977 & 0.3846656 & 40.3210545 & 199.9802367 & 5885.4032828 \\
\hline & Present Study & $\begin{array}{c}0.778414566493 \\
5\end{array}$ & $\begin{array}{c}0.38478017 \\
10354\end{array}$ & $\begin{array}{c}40.33193039 \\
98310\end{array}$ & $\begin{array}{c}199.830393131 \\
8342\end{array}$ & 5596.186418 \\
\hline \multirow{6}{*}{ II } & Dimopoulos (2007) & 0.75 & 0.375 & 38.86010 & 221.36549 & 5850.38306 \\
\hline & Mahdavi et al. (2007) & 0.75 & 0.375 & 38.86010 & 221.36553 & 5849.76169 \\
\hline & Hedar and Fukushima (2006) & 0.7683257 & 0.3797837 & 39.8096222 & 207.2255595 & 5868.764836 \\
\hline & Gandomi et al. (2011a) & 0.75 & 0.375 & 38.86010 & 221.36547 & 5850.38306 \\
\hline & Garg (2014) & 0.7275958 & 0.3596552 & 37.6991359 & 239.999805 & 5804.448670 \\
\hline & Present Study & $\begin{array}{c}0.728093172620 \\
1\end{array}$ & $\begin{array}{c}\mathbf{0 . 3 5 9 9 2 2 5 4} \\
\mathbf{3 3 9 3 7}\end{array}$ & $\begin{array}{c}\text { 37.72416652 } \\
\mathbf{4 8 2 8 5}\end{array}$ & $\begin{array}{c}239.585763017 \\
3754\end{array}$ & 5501.57955 \\
\hline
\end{tabular}

Table 2. Statistical results of different methods for pressure vessel problem

\begin{tabular}{|c|c|c|c|c|c|c|}
\hline Version & Method & Best & Mean & Worst & Std. Dev. & Median \\
\hline \multirow{15}{*}{ I } & Sandgren (1998) & 8129.1036 & NA & NA & NA & NA \\
\hline & Kannan and Kramer (1994) & 7198.0428 & NA & NA & NA & NA \\
\hline & Deb (1997) & 6410.3811 & NA & NA & NA & NA \\
\hline & Coello (2000) & 6288.7445 & 6293.8432 & 6308.1497 & 7.4133 & NA \\
\hline & Coello and Montes (2002) & 6059.9463 & 6177.2533 & 6469.3220 & 130.9297 & NA \\
\hline & He and Wang (2007) & 6061.0777 & 6147.1332 & 6363.8041 & 86.4545 & NA \\
\hline & Montes and Coello (2008) & 6059.7456 & 6850.0049 & 7332.8798 & 426.0000 & NA \\
\hline & Kaveh and Talatahari (2009) & 6059.7258 & 6081.7812 & 6150.1289 & 67.2418 & NA \\
\hline & Kaveh and Talatahari (2010) & 6059.0925 & 6075.2567 & 6135.3336 & 41.6825 & NA \\
\hline & Gandomi et al. (2003) & 6059.714 & 6447.7360 & 6495.3470 & 502.693 & NA \\
\hline & Cagnina et. al. (2008) & 6059.714335 & 6092.0498 & NA & 12.1725 & NA \\
\hline & Dos Santos Coelho (2010) & 6059.7208 & 6440.3786 & 7544.4925 & 448.4711 & 6257.5943 \\
\hline & He et al. (2004) & 6059.7143 & 6289.92881 & NA & 305.78 & NA \\
\hline & Akay and Karaboga (2012) & 6059.714339 & 6245.308144 & NA & 205 & NA \\
\hline & Garg (2014) & 5885.40328280 & 5887.5570240 & 5895.126804 & 2.745290 & 5886.149289 \\
\hline \multirow{7}{*}{ II } & Present Study & 5596.18642 & 5599.28868 & 5648.15212 & 11.5203421 & 5596.44649 \\
\hline & Dimopoulos (2007) & 5850.38306 & NA & NA & NA & NA \\
\hline & Mahdavi et al. (2007) & 5849.7617 & NA & NA & NA & NA \\
\hline & Hedar and Fukushima (2006) & 5868.764836 & 6164.585867 & 6804.328100 & 257.473670 & NA \\
\hline & Gandomi et al. (2011a) & 5850.38306 & 5937.33790 & 6258.96825 & 164.54747 & NA \\
\hline & $\operatorname{Garg}(2014)$ & 5804.4486708 & 5805.4739140 & 5811.977127 & 1.41146216 & 5805.0737979 \\
\hline & Present Study & 5501.57955 & 5504.44726 & 5524.91931 & 5.79839866 & 5502.342012 \\
\hline
\end{tabular}


International Journal of Mathematical, Engineering and Management Sciences

Vol. 4, No. 4, 968-981, 2019

https://dx.doi.org/10.33889/IJMEMS.2019.4.4-077

\subsection{Design of Welded Beam}

The objective of this design problem is to minimize the cost of fabrication of welded beam given the constraints on shear stress, bending stress in the beam, buckling load on the bar, end deflection of the beam and side constraints. The variables in the problem are thickness of the weld $h=x_{1}$, length of joint $l=x_{2}$, width of the beam $t=x_{3}$, thickness of beam $b=x_{4}$. The problem has been taken from Rao (1996).

Version 1: The fitness function is given by:

Minimize $f(X)=1.1047 x_{1}^{2} x_{2}+0.04811 x_{3} x_{4}\left(14+x_{2}\right)$

subject to:

$$
\begin{aligned}
& g_{1}(X)=T(X)-T_{\max } \leq 0, g_{2}(X)=\sigma(X)-\sigma_{\max } \leq 0, g_{3}(X)=x_{1}-x_{4} \leq 0, \\
& g_{4}(X)=0.125-x_{1} \leq 0, g_{5}(X)=\delta(X)-0.25 \leq 0, g_{6}(X)=P-P_{c}(X) \leq 0, \\
& 0.1 \leq x_{1} \leq 2 ; 0.1 \leq x_{2} \leq 10 ; 0.1 \leq x_{3} \leq 10 ; 0.1 \leq x_{4} \leq 2 .
\end{aligned}
$$

where shear stress within the weld is given by $T$, allowable shear stress which is equal to 13600 psi is given by $T_{\max }$, the normal stress within the beam which is equal to 30000 psi is given by $\sigma_{\text {max }}$, whereas the buckling load of the bar is given by $P_{c}$, the load equal to $6000 \mathrm{lb}$ is given by $P$ and the end deflection is noted by $\delta$.

$T(X)=T_{1}^{2}+2 T_{1} T_{2}\left(\frac{x^{2}}{2 R}\right)+r_{2}^{2}$ where $T_{1}=\frac{P}{\sqrt{2} x_{1} x_{2}}, T_{2}=\frac{M R}{J}$,

where $M=P\left(L+\frac{x_{2}}{2}\right), J(X)=2\left[\frac{x_{1} x_{2}}{\sqrt{2}}\left\{\frac{x_{2}^{2}}{12}+\left(\frac{x_{1}+x_{3}}{2}\right)^{2}\right\}\right], R=\sqrt{\frac{x_{2}^{2}}{4}+\left(\frac{x_{1}+x_{3}}{2}\right)^{2}}$,

$\sigma(X)=\frac{6 P L}{x_{4} x_{3}^{2}}, \delta(X)=\frac{4 P L^{3}}{E x_{3}^{3} x_{4}}, P_{c}(X)=\frac{4.013 \sqrt{\frac{E G x_{3}^{2} x_{4}^{6}}{36}}}{L^{2}}\left(1-\frac{x_{3}}{2 L} \sqrt{\frac{E}{4 G}}\right)$.

$G=12 \times 10^{6} \mathrm{psi}, E=30 \times 10^{6} \mathrm{psi}, P=6000 \mathrm{lb}, \mathrm{L}=14 \mathrm{inch}$.

The solution obtained for this problem using crow search algorithm:

$X=(0.244343277069188,6.213952517706234,8.296218570994581,0.244345831688385)$.

Optimum (minimum) fitness function value: $f(X)=2.38122873948154$.

Constraints value:

$G=\left(g_{1}, g_{2}, g_{3}, g_{4}, g_{5}, g_{6}\right)=(-8402.2936827306,-23694.6260885614,-0.2081091708$, - 0.8905141940, - 0.2466020386, - 733939.8720985127). 
International Journal of Mathematical, Engineering and Management Sciences

Vol. 4, No. 4, 968-981, 2019

https://dx.doi.org/10.33889/IJMEMS.2019.4.4-077

A comparison with other algorithms has been shown in the Table 3 and it found that the results obtained using proposed algorithms are better than existing results.

\section{Version 2:}

In this version a new constraint, along with the constraints used in version 1 , has been added which includes deflection, polar moment of inertia and buckling load.

$$
\begin{aligned}
& g_{7}(X)=0.10471 x_{1}^{2}+0.04811 x_{3} x_{4}\left(14+x_{2}\right)-5 \leq 0, \quad \delta(X)=\frac{6 P L^{3}}{E x_{3}^{2} x_{4}} \\
& P_{c}(X)=\frac{4.013 E \sqrt{\frac{x_{3}^{2} x_{4}^{6}}{36}}}{L^{2}}\left(1-\frac{x_{3}}{2 L} \sqrt{\frac{E}{4 G}}\right), J(X)=2\left[\sqrt{2} x_{1} x_{2}\left\{\frac{x_{2}^{2}}{4}+\left(\frac{x_{1}+x_{3}}{2}\right)^{2}\right\}\right] .
\end{aligned}
$$

The solution obtained for this problem using proposed algorithm:

$X=(0.205671231425633,3.254535728596008,9.036762134420712,0.205729413100657)$.

Optimum (minimum) fitness function value: $f(X)=1.69527215466093$.

Constraints values:

$G=\left(g_{1}, g_{2}, g_{3}, g_{4}, g_{5}, g_{6}, g_{7}\right)=(-2650.9695371295,-13840.0862642908,-0.4891852108$,

$-0.7480686955,-0.0838556961,-0.2342050396,-182819.6954371415)$.

A comparison with other algorithms has been shown in the Table 3 and it is found that the results obtained using proposed algorithms are better than other algorithms. Further, statistical analysis has been done for version 1 and version 2 and a comparison with other algorithms has been shown in Table 4. 
International Journal of Mathematical, Engineering and Management Sciences

Vol. 4, No. 4, 968-981, 2019

https://dx.doi.org/10.33889/IJMEMS.2019.4.4-077

Table 3. Comparison of the best solution for welded beam design problem obtained by different methods

(NA means not available)

\begin{tabular}{|c|c|c|c|c|c|c|}
\hline \multirow[t]{2}{*}{ Version } & \multirow[t]{2}{*}{ Method } & \multicolumn{4}{|c|}{ Design Variables } & \multirow[t]{2}{*}{$f(x)$} \\
\hline & & $x_{1}$ & $x_{2}$ & $x_{3}$ & $x_{4}$ & \\
\hline \multirow{10}{*}{ I } & Ragsdell and Phillips (1976) & 0.245500 & 6.196000 & 8.273000 & 0.245500 & 2.385937 \\
\hline & Rao (1996) & 0.245500 & 6.196000 & 8.273000 & 0.245500 & 2.3860 \\
\hline & Deb (1991) & 0.248900 & 6.173000 & 8.178900 & 0.253300 & 2.433116 \\
\hline & Deb (2000) & NA & NA & NA & NA & 2.38119 \\
\hline & Ray and Liew (2003) & 0.244438276 & 6.237967234 & 8.288576143 & 0.2445661820 & 2.3854347 \\
\hline & Lee and Geem (2005) & 0.2442 & 0 & 0 & 0.2443 & 2.38 \\
\hline & Hwang and $\mathrm{He}(2006)$ & 0.223100 & 6.2231 & 8.2915 & 0.2245 & 2.25 \\
\hline & Mehta and Dasgupta (2012) & 0.24436895 & 1.5815 & 12.84680 & 0.24436895 & 2.3811341 \\
\hline & Garg (2014) & 0.24436198 & 6.21767407 & 8.29163558 & 0.24436883 & 2.38099617 \\
\hline & Present Study & $\begin{array}{c}0.244343277 \\
069188\end{array}$ & $\begin{array}{c}6.213952517 \\
706234\end{array}$ & $\begin{array}{c}8.296218570 \\
994581\end{array}$ & $\begin{array}{c}.24434583168 \\
8385 \\
\end{array}$ & 2.381228739 \\
\hline \multirow{18}{*}{ II } & Coello (2000) & 0.208800 & 3.420500 & 8.997500 & 0.210000 & 1.748309 \\
\hline & Coello and Montes (2002) & 0.205986 & 3.471328 & 9.020224 & 0.206480 & 1.728226 \\
\hline & Hu et al. (2003) & 0.20573 & 3.47049 & 9.03662 & 0.20573 & 1.72485084 \\
\hline & Hedar and Fukushima (2006) & 0.205644261 & 3.472578742 & 9.03662391 & 0.2057296 & 1.7250022 \\
\hline & He and Wang (2007) & 0.202369 & 3.544214 & 9.048210 & 0.205723 & 1.728024 \\
\hline & Dimopoulos (2007) & 0.2015 & 3.5620 & 9.041398 & 0.205706 & 1.731186 \\
\hline & Mahdavi et al. (2007) & 0.20573 & 3.47049 & 9.03662 & 0.20573 & 1.7248 \\
\hline & Montes et al. (2007) & 0.205730 & 3.470489 & 9.036624 & 0.205730 & 1.724852 \\
\hline & Montes and Coello (2008) & 0.199742 & 3.612060 & 9.037500 & 0.206082 & 1.73730 \\
\hline & Cagnina et al. (2008) & 0.205729 & 3.470488 & 9.036624 & 0.205729 & 1.724852 \\
\hline & Fesanghary et al. (2008) & 0.20572 & 3.47060 & 9.03682 & 0.20572 & 1.7248 \\
\hline & Kaveh and Talatahari (2009) & 0.205729 & 3.469875 & 9.036805 & 0.205765 & 1.724849 \\
\hline & Kaveh and Talatahari (2010) & 0.205700 & 3.471131 & 9.036683 & 0.205731 & 1.724918 \\
\hline & Gandomi et al. (2011a) & 0.2015 & 3.562 & 9.0414 & 0.2057 & 1.73121 \\
\hline & Mehta and Dasgupta (2012) & 0.20572885 & 3.47050567 & 9.03662392 & 0.20572964 & 1.724855 \\
\hline & Akay and Karaboga (2012) & 0.205730 & 3.470489 & 9.036624 & 0.205730 & 1.724852 \\
\hline & Garg (2014) & 0.20572450 & 3.25325369 & 9.03664438 & 0.20572999 & 1.69526388 \\
\hline & Present Study & $\begin{array}{c}0.205671231 \\
425633\end{array}$ & $\begin{array}{c}3.254535728 \\
596008\end{array}$ & $\begin{array}{c}9.036762134 \\
420712\end{array}$ & $\begin{array}{c}0.20572941310 \\
0657\end{array}$ & 1.695272155 \\
\hline
\end{tabular}

Table 4. Statistical results of different methods for welded beam design problem

\begin{tabular}{|c|l|c|c|c|c|c|}
\hline Version & Method & Best & Mean & Worst & Std. Dev. & Median \\
\hline \multirow{6}{*}{ I } & Regsdell and Phillips (1976) & 2.385937 & N A & N A & N A & N A \\
& Rao (1996) & 2.3860 & N A & N A & N A & N A \\
& Deb (1991) & 2.433116 & N A & N A & N A & N A \\
& Deb (2000) & 2.38119 & N A & N A & NA & N A \\
& Ray and Liew (2003) & 2.3854347 & 3.2551371 & 6.3996785 & 0.9590780 & 3.0025883 \\
& Lee and Geem (2005) & 2.38 & N A & N A & N A & N A \\
& Hwang and He (2006) & 2.25 & 2.26 & 2.28 & N A & N A \\
& Mehta and Dasgupta (2012) & 2.381134 & 2.3811786 & 2.3812614 & NA & 2.3811641 \\
& Garg (2014) & 2.38099617 & 2.38108932 & 2.38146999 & $1.01227 \mathrm{E}-4$ & 2.38107233 \\
\hline \multirow{6}{*}{ Present Study } & $\mathbf{2 . 3 8 1 2 2 8 7 4}$ & $\mathbf{2 . 3 8 1 4 1 6 3 7}$ & $\mathbf{2 . 3 8 2 3 2 9 1 5}$ & $\mathbf{0 . 0 0 0 3 6 6 7 6}$ & $\mathbf{2 . 3 8 1 2 9 9 1 0 6}$ \\
\hline & Coello (2000) & 1.748309 & 1.771973 & 1.785835 & 0.011220 & N A \\
& Coello and Montes (2002) & 1.728226 & 1.792654 & 1.993408 & 0.07471 & N A \\
& Dimopoulos (2007) & 1.731186 & N A & N A & N A & N A \\
& He and Wang (2007) & 1.728024 & 1.748831 & 1.782143 & 0.012926 & N A \\
& Hedar and Fukushima (2006) & 1.7250022 & 1.7564428 & 1.8843960 & 0.0424175 & N A \\
& Montes et. al. (2007) & 1.724852 & 1.725 & N A & 1 E-15 & N A \\
& Montes and Coello (2008) & 1.737300 & 1.813290 & 1.994651 & 0.070500 & N A \\
& Cagnina et al. (2008) & 1.724852 & 2.0574 & N A & 0.2154 & N A \\
& Kaveh and Talatahari (2010) & 1.724918 & 1.729752 & 1.775961 & 0.009200 & N A \\
& Kaveh and Talatahari (2009) & 1.724849 & 1.727564 & 1.759522 & 0.008254 & N A \\
& Gandomi et al. (2011a) & 1.7312065 & 1.8786560 & 2.3455793 & 0.2677989 & N A \\
& Mehta and Dasgupta (2012) & 1.724855 & 1.724865 & 1.72489 & N A & 1.724861 \\
& Akay and Karaboga (2012) & 1.724852 & 1.741913 & N A & 0.031 & N A \\
& Garg (2014) & 1.69526388 & 1.69530842 & 1.69537060 & $2.836238 E-5$ & 1.69530879 \\
\hline & Present Study & $\mathbf{1 . 6 9 5 2 7 2 1 5}$ & $\mathbf{1 . 6 9 5 3 4 0 9}$ & $\mathbf{1 . 6 9 5 5 1 8 7 9}$ & $\mathbf{6 . 9 9 9 6 E - 0 5}$ & $\mathbf{1 . 6 9 5 3 2 3 7 9 2}$ \\
\hline
\end{tabular}


International Journal of Mathematical, Engineering and Management Sciences

Vol. 4, No. 4, 968-981, 2019

https://dx.doi.org/10.33889/IJMEMS.2019.4.4-077

\subsection{Design of Tension / Compression String}

Tension/Compression string problem requires to optimize (minimize) the weight of the string and it has been given by Belegundu (1982) and Arora (1989). The string is constrained to minimum deflection, constrained diameter, shear stress etc. The variables that we have to optimize are coil diameter $\left(x_{1}\right)$, the wire diameter $\left(x_{2}\right)$ and the number of coil $\left(x_{3}\right)$.

The fitness function is given by: Minimize $f(X)=\left(x_{3}+2\right) x_{2} x_{1}^{2}$

$$
\begin{aligned}
& \text { subject to : } g_{1}(X)=1-\frac{x_{2}^{3} x_{3}}{71785 x_{1}^{4}} \leq 0, g_{2}(X)=\frac{4 x_{2}^{2}-x_{1} x_{2}}{12566\left(x_{2} x_{1}^{3}-x_{1}^{4)}\right.}+\frac{1}{5108 x_{1}^{2}}-1 \leq 0, \\
& g_{3}(X)=1-\frac{140.45 x_{1}}{x_{2}^{2} x_{3}} \leq 0, g_{4}(X)=\frac{x_{1}+x_{2}}{1.5}-1 \leq 0 . \\
& 0.05 \leq x_{1} \leq 2 ; 0.25 \leq x_{2} \leq 1.3 ; 2 \leq x_{3} \leq 15 .
\end{aligned}
$$

The solution obtained for this problem using crow search algorithm:

$$
X=(0.051686690341221,0.356658764326658,11.292540928654185) .
$$

Optimum (minimum) fitness function value: $f(X)=0.012665248980102$.

Constraints value:

$$
\begin{gathered}
G=\left(g_{1}, g_{2}, g_{3}, g_{4}\right)=(-0.610136631957694,-0.093497586159253,-1.953085401792193, \\
-0.623891361731687) .
\end{gathered}
$$

The results obtained using the proposed algorithms are found to be better than the other algorithms (See Table 5). Further, statistical analysis has been done and a comparison with other algorithms has been shown in the Table 6 . 
International Journal of Mathematical, Engineering and Management Sciences

Vol. 4, No. 4, 968-981, 2019

https://dx.doi.org/10.33889/IJMEMS.2019.4.4-077

Table 5. Comparison of the best solution for tension/compression string design problem by different methods

\begin{tabular}{|l|c|c|c|c|}
\hline \multirow{2}{*}{ Method } & \multicolumn{3}{|c|}{ Design Variables } & \multirow{2}{*}{$\boldsymbol{f ( x )}$} \\
\cline { 2 - 4 } & $\boldsymbol{x}_{\mathbf{1}}$ & $\boldsymbol{x}_{\mathbf{2}}$ & $\boldsymbol{x}_{\mathbf{3}}$ & 0.0128334 \\
Belegundu (1982) & 0.05 & 0.315900 & 14.25000 & 0.0127303 \\
Arora (1989) & 0.053396 & 0.399180 & 185400 & 0.01270478 \\
Coello (2000) & 0.051480 & 0.351661 & 1.632201 & 0.013060 \\
Ray and Saini (2001) & 0.050417 & 0.321532 & 13.979915 & 0.0126810 \\
Coello and Montes (2002) & 0.051989 & 0.363965 & 10.890522 & 0.1266924934 \\
Ray and Liew (2003) & 0.0521602170 & 0.368158695 & 10.6484422590 & $0.0126661409^{*}$ \\
Hu et al. (2003) & 0.051466369 & 0.351383949 & 11.60865920 & $0.0126652812^{*}$ \\
He et al. (2004) & 0.05169040 & 0.35674999 & 11.28712599 & 0.012665285 \\
Hedar and Fukushima (2006) & 0.05174250340926 & 0.35800478345599 & 11.21390736278739 & 0.01274840 \\
Raj et al. (2005) & 0.05386200 & 0.41128365 & 0.68437980 & 0.01266523 \\
Tsai (2005) & 0.05168906 & 0.3567178 & 11.28896 & 0.0126706 \\
Mahdavi et al. (2007) & 0.05115438 & 0.34987116 & 12.0764321 & 0.012665 \\
Montes et al. (2007) & 0.051688 & 0.356692 & 11.290483 & 0.0126747 \\
He and Wang (2007) & 0.051728 & 0.357644 & 11.244543 & 0.012655 \\
Cagnina et al. (2008) & 0.051583 & 0.354190 & 11.438675 & 0.012665233 \\
Zhang et al. (2008) & 0.0516890614 & 0.3567177469 & 11.2889653382 & 0.012698 \\
Montes and Coello (2008) & 0.051643 & 0.355360 & 11.397926 & 0.0126652375 \\
Omran and Salman (2009) & 0.0516837458 & 0.3565898352 & 11.2964717107 & $0.0126432 *$ \\
Keveh and Talatahari (2010) & 0.051865 & 0.361500 & 11.0000 & 0.012665 \\
Dos Santos Coelho (2010) & 0.051515 & 0.352529 & 11.538862 & 0.012665 \\
Akay and Karaboga (2012) & 0.051749 & 0.358179 & 11.203763 & 0.0126652327883 \\
Garg (2014) & 0.051689156131 & 0.356720026419 & 11.288831695483 & $\mathbf{0 . 0 1 2 6 6 5 2 4 9}$ \\
\hline Present Study & $\mathbf{0 . 0 5 1 6 8 6 6 9 0 3 4 1 2 2 1}$ & $\mathbf{0 . 3 5 6 6 5 8 7 6 4 3 2 6 6 5 8}$ & $\mathbf{1 1 . 2 9 2 5 4 0 9 2 8 6 5 4 1 8 5}$ & $\mathbf{0}$ \\
\hline
\end{tabular}

*Infeasible solution as they violate one of the constraints set.

Table 6. Statistical results of different methods for tension / compression string problem (NA means not available)

\begin{tabular}{|c|c|c|c|c|c|}
\hline Method & Best & Mean & Worst & Std. Dev. & Median \\
\hline Belegundu (1982) & 0.0128334 & NA & NA & NA & NA \\
\hline Arora (1989) & 0.0127303 & NA & NA & NA & NA \\
\hline Coello (2000) & 0.01270478 & 0.01276920 & 0.01282208 & $3.9390 \times 10^{-5}$ & 0.01275576 \\
\hline Ray and Saini (2001) & 0.0130600 & 0.015526 & 0.018992 & NA & NA \\
\hline Coello and Montes (2002) & 0.0126810 & 0.012742 & 0.012973 & $5.9000 \times 10^{-5}$ & NA \\
\hline Ray and Liew (2003) & 0.01266924934 & 0.012922669 & 0.016717272 & $5.92 \times 10^{-4}$ & 0.012922669 \\
\hline Hu et al. (2003) & 0.0126661409 & 0.012718975 & NA & $6.446 \times 10^{-5}$ & NA \\
\hline He et al. (2004) & 0.0126652812 & 0.01270233 & NA & $4.12439 \times 10^{-5}$ & NA \\
\hline He and Wang. (2007) & 0.0126747 & 0.012730 & 0.012924 & $5.1985 \times 10^{-5}$ & NA \\
\hline Zhang et al. (2008) & 0.012665233 & 0.012669366 & 0.012738262 & $1.25 \times 10^{-5}$ & NA \\
\hline Hedar and Fukushima (2006) & 0.012665285 & 0.012665299 & 0.012665338 & $2.2 \times 10^{-8}$ & NA \\
\hline Montes et al. (2007) & 0.012665 & 0.012666 & NA & $2.0 \times 10^{-6}$ & NA \\
\hline Montes and Coello (2008) & 0.012698 & 0.013461 & 0.164850 & $9.6600 \times 10^{-4}$ & NA \\
\hline Cagnina et al. (2008) & 0.012665 & 0.0131 & NA & $4.1 \times 10^{-4}$ & NA \\
\hline Kaveh and Talatahari (2010) & 0.0126432 & 0.012720 & 0.012884 & $3.4888 \times 10^{-5}$ & NA \\
\hline Omran and Salman (2009) & 0.0126652375 & 0.0126652642 & NA & NA & NA \\
\hline Dos Santos Coelho (2010) & 0.012665 & 0.013524 & 0.017759 & 0.001268 & 0.012957 \\
\hline Akay and Karaboga (2012) & 0.012665 & 0.012709 & NA & 0.012813 & NA \\
\hline Garg (2014) & 0.0126652327883 & 0.0126689724845 & 0.012710407729 & $9.429426 \mathrm{E}-6$ & 0.12665314728 \\
\hline Present Study & 0.01266525 & 0.01266604 & 0.01267091 & 1.5293E-06 & 0.012665449 \\
\hline
\end{tabular}


International Journal of Mathematical, Engineering and Management Sciences

Vol. 4, No. 4, 968-981, 2019

https://dx.doi.org/10.33889/IJMEMS.2019.4.4-077

\section{Conclusions}

A crow search algorithm is applied to various structural engineering design problems such as Pressure vessel design optimization, Welded beam design optimization, Tension/Compression string design optimization. The numerical results are compared with the various existing optimization algorithms including metaheuristic algorithms and it is found that the results obtained by the crow search algorithm are better than others. Further, the effectiveness of the algorithm is verified to be better than the existing algorithms by statistical analysis using mean, median, best case, worst case scenarios and it is observed that crow search algorithm is better than other existing algorithms. It is also remarked that the standard deviation has been found to be pretty less than that by others method. The present study confirms that the crow search algorithm may be easily and effectively applied to various structural engineering design problems.

On observing the performance of crow search, this paper opens doors for new research directions in crow search algorithm. The power of the algorithm suggests that the crow search should be implemented in integer optimization problems and multi-objective optimization problems. Further, it can also be parallelized for solving large scale problems.

\section{Conflict of Interest}

The author confirms that there is no conflict of interest to declare for this publication.

\section{Acknowledgment}

The author would like to thank the learned reviewers for his/her valuable comments to improve the quality of the paper.

\section{References}

Akay, B., \& Karaboga, D. (2012). Artificial bee colony algorithm for large-scale problems and engineering design optimization. Journal of Intelligent Manufacturing, 23(4), 1001-1014.

Arora, J.S. (1989). Introduction to optimum design, $3^{\text {rd }}$ edition. Academic Press, Elsevier, USA.

Askarzadeh, A. (2016). A novel metaheuristic method for solving constrained engineering optimization problems, Crow search algorithm. Computers \& Structures, 169, 1-12.

Belegundu, A.D. (1982). A study of mathematical programming methods for structural optimization, $P h D$ thesis, Department of Civil and Environmental Engineering. University of Iowa, Iowa, USA.

Cagnina, L.C, Esquivel, S.C., \& Coello, C.A.C. (2008). Solving engineering optimization problem with the simple constrained particle swarm optimizer. Informatica, 32(3), 319-326.

Coello, C.A.C. (2000). Use of a self -adaptive penalty approach for engineering optimization problems. Computers in Industry, 41(2), 113-127.

Coello, C.A.C., \& Montes, E.M. (2002). Constraint- handling in genetic algorithms through the use of dominance-based tournament selection. Advanced Engineering Informatics, 16(3), 193-203.

Deb, K. (1991). Optimal design of a welded beam via genetic algorithms. AIAA Journal, 29(11), 20132015. 
International Journal of Mathematical, Engineering and Management Sciences

Vol. 4, No. 4, 968-981, 2019

https://dx.doi.org/10.33889/IJMEMS.2019.4.4-077

Deb, K. (1997). GeneAS: A robust optimal design technique for mechanical component design. In: Evolutionary Algorithms in Engineering Applications, Springer, Berlin, Heidelberg, 497-514.

Deb, K. (2000). An efficient constraint handling method for genetic algorithms. Computer Methods in Applied Mechanics and Engineering, 186, 311-338.

Dimopoulos, G.G. (2007). Mixed-variable engineering optimization based on evolutionary and social metaphors. Computer Methods in Applied Mechanics and Engineering, 196(4-6), 803-817.

dos Santos Coelho, L. (2010). Gaussian quantum-behaved particle swarm optimization approaches for constrained engineering design problems. Expert Systems with Applications, 37(2), 1676-1683.

Fesanghary, M., Mahdavi, Minary-Jolandan, M., \& Alizadeh, Y. (2008). Hybridizing harmony search algorithm with sequential quadratic programming for engineering optimization problems. Computer Methods in Applied Mechanics and Engineering, 197(33-40), 3080-3091.

Gandomi, A.H, Yang, X.S., \& Alavi, A.H. (2011). Mixed variable structural optimization using firefly algorithm. Computers \& Structures, 89(23-24), 2325-2336.

Gandomi, A.H., Yang, X.S., \& Alavi, A.H. (2003). Cuckoo search algorithm: a metaheuristic approach to solve structural optimization problems. Engineering with Computers, 29 (1), 17-35.

Garg, H. (2014). Solving structural engineering design optimization problems using an artificial bee colony algorithm. Journal of Industrial and Management Optimization, 10(3), 777-794.

Geem Z.W., Kim J.H., \& Loganathan G.V. (2001). A new heuristic optimization algorithm: harmony search, Simulation, 76(2), 60-68.

Glover F. (1986). Future paths for integer programming and links to artificial intelligence. Computers and Operations Research, 13(5), 533-549.

He, Q., \& Wang, L. (2007). An effective co - evolutionary particle swarm optimization for constrained engineering design problems. Engineering Applications of Artificial Intelligence, 20(1), 89-99.

He, S., Prempain, E., \& Wu, Q.H (2004). An improved particle swarm optimizer for mechanical design optimization problems. Engineering Optimization, 36(5), 585-605.

Hedar, A.R., \& Fukushima, M. (2006). Derivative - free filter simulated annealing method for constrained continuous global optimization. Journal of Global Optimization, 35(4), 521-549.

Holland, J.H. (1975). Adaptation in natural and artificial systems Ann Arbor. The University of Michigan Press, $1,975$.

Hu, X.H., Eberhart, R.C., \& Shi, Y.H. (2003). Engineering optimization with particle swarm. Proceedings of the 2003 IEEE Swarm Intelligence Symposium, 53-57.

Hwang, S.F., \& He, R.S. (2006). A hybrid real-parameter genetic algorithm for function optimization. Advanced Engineering Informatics, 20(1), 7-21.

Kannan, B.K., \& Kramer, S.N. (1994). An augmented Lagrange multiplier based method for mixed integer discrete continuous optimization and its applications to mechanical design. Journal of Mechanical Design, 116(2), 405-411.

Kaveh, A., \& Talatahari, S. (2009). Engineering optimization with hybrid particle swarm and ant colony optimization. Asian Journal of Civil Engineering (Building and Housing), 10(6), 611-628.

Kaveh, A., \& Talatahari, S. (2010). An improved ant colony optimization for constrained engineering design problems. Engineering Computations, 27(1), 155-182.

Kennedy, J., \& Eberhart, R.C. (1995). Particle swarm optimization. Proceedings of the IEEE International Conference on Neural Networks, 4, 1942-1948. 
International Journal of Mathematical, Engineering and Management Sciences

Vol. 4, No. 4, 968-981, 2019

https://dx.doi.org/10.33889/IJMEMS.2019.4.4-077

Lee, K.S., \& Geem, Z.W. (2005). A new meta-heuristic algorithm for continuous engineering optimization: harmony search theory and practice. Computer Methods in Applied Mechanics and Engineering, 194(36-38), 3902-3933.

Mahdavi, M., Fesanghary, M., \& Damangir, E. (2007). An improved harmony search algorithm for solving optimization problems. Applied Mathematics and Computation, 188(2), 1567-1579.

Mehta, V.K., \& Dasgupta, B. (2012). A constrained optimization algorithm based on the simplex search method. Engineering Optimization, 44(5), 537-550.

Mezura-Montes, E., \& Coello, C.A.C. (2008). An empirical study about the usefulness of evolution strategies to solve constrained optimization problems. International Journal of General Systems, 37(4), 443-473.

Mezura-Montes, E., Coello Coello, C.A., Velázquez-Reyes, J., \& Muñoz-Dávila, L. (2007). Multiple trial vectors in differential evolution for engineering design. Engineering Optimization, 39(5), 567-589.

Michalewicz, Z. (1994). Genetic Algorithms+Data Structures = Evolution Programs, Springer -Verlag, Berlin.

Mirjalili S., Gandomi, A.H., Mirjalili, S.Z., Saremi, S., Faris, H., \& Mirjalili, S.H. (2017). Salp Swarm Algorithm: A bio-inspired optimizer for engineering design problems. Advances in Engineering Software, 114, 163-191.

Omran, M.G.H., \& Salman, A. (2009). Constrained optimization using CODEQ. Chaos, Solitons \& Fractals, 42(2), 662-668.

Ragsdell, K.M., \& Phillips, D.T. (1976). Optimal design of a class of welded structures using geometric programming. Journal of Engineering for Industry, 98(3), 1021-1025.

Raj, K.H., Sharma, R.S., Mishra, G.S., Dua, A., \& Patvardhan, C. (2005). An evolutionary computational technique for constrained optimization in engineering design. Journal of the Institution of Engineers India Part: Mechanical Engineering Division, 86, 121-128.

Rao, S.S. (1996). Engineering optimization: theory and practice, 3rd edition, John Wiley \& Sons, Chichester.

Ray, T., \& Liew, K.M. (2003). Society and civilization: An optimization algorithm based on the simulation of social behavior. IEEE Transactions on Evolutionary Computation, 7(4), 386- 396.

Ray, T., \& Saini, P. (2001). Engineering design optimization using a swarm with intelligent information sharing among individuals. Engineering Optimization, 33(6), 735-748.

Safiye T. (2018). Multi Objective Simulated Annealing Approach for Facility Layout Design. International Journal of Mathematical, Engineering and Management Sciences, 3(4), 365-380.

Sandgren, E. (1988). Nonlinear integer and discrete programming in mechanical design, Proceedings of the ASME Design Technology Conference, F.L. Kissimine, 95-105.

Shi, Y., \& Eberhart, R. (1998). A modified particle swarm optimizer. In: 1998 IEEE International Conference on Evolutionary Computation Proceedings. IEEE World Congress on Computational Intelligence, 69-73.

Tsai, J. (2005). Global optimization of nonlinear fractional programming problems in engineering design. Engineering Optimization, 37(4), 399-409.

Yang X.S. (2011). Metaheuristic Optimization. Scholar Pedia, 6(8), 11472.

Yang, X.S., \& Deb, S. (2009). Cuckoo search via Lévy flights. In: Proceedings of World Congress on Nature \& Biologically Inspired Computing (NaBIC 2009), December 2009, India. IEEE Publications, USA, 210-214. 
International Journal of Mathematical, Engineering and Management Sciences

Vol. 4, No. 4, 968-981, 2019

https://dx.doi.org/10.33889/IJMEMS.2019.4.4-077

Zhang, C., \& Wang, H.P. (1993). Mixed-discrete nonlinear optimization with simulated annealing. Engineering Optimization, 21(4), 277-291.

Zhang, M., Luo, W., \& Wang, X. (2008). Differential evolution with dynamic stochastic selection for constrained optimization. Information Sciences, 178(15), 3043-3074.

Zolghadr-Asli, B., Bozorg-Haddad, O., \& Chu, X. (2018). Crow search algorithm (CSA). In: Advanced Optimization by Nature-Inspired Algorithms, Springer, Singapore, 143-149.

(c) (7) Original content of this work is copyright () International Journal of Mathematical, Engineering and Management Sciences. All rights reserved. Except of uses under a Creative Commons Attribution 4.0 International (CC BY 4.0) license at https://creativecommons.org/licenses/by/4.0/ 